\title{
COVID - 19 complicated by Acute Respiratory Distress Syndrome, Myocarditis, and Pulmonary Embolism. A case report
}

\author{
Rajai F. Bulbul, Jassim Al Suwaidi*, Mohammed Al-Hijji, Hassan Al Tamimi, Ibrahim Fawzi \\ Hamad Medical Corporation, Doha, Qatar
}

\begin{abstract}
A 49-year-old female Qatari woman, with no past medical history, presented at a hospital complaining of a history of cough and shortness of breath. The patient tested positive for severe acute respiratory syndrome (ARDS) and COVID-19. Subsequently, her course of treatment was complicated by severe acute respiratory distress syndrome, pulmonary embolism and severe myocarditis requiring treatment with venous-arterial extracorporeal membrane oxygenation as a bridge to complete recovery.
\end{abstract}

Keywords: COVID-19, SARS-CoV-2, myocarditis, cardiac injury, extracorporeal membrane oxygenation, acute respiratory distress syndrome (ARDS), cardiogenic shock

Received: 29 July 2020 / Accepted: 30 October 2020

\section{BACKGROUND}

In December 2019, coronavirus disease 2019 (COVID-19) was first described in Wuhan, China, in patients complaining of flu-like symptoms. The mortality rate of COVID-19 disease, estimated to be $<1 \%$, is a result of patients developing severe acute respiratory syndrome (ARDS) and multi-organ dysfunction. A minority of patients develop acute cardiovascular manifestations, including myocardial infarction and myocarditis-like syndrome [1-11]. In this report, a case of a young, healthy Qatari woman who developed COVID-19 myocarditis requiring treatment with venous-arterial extracorporeal membrane oxygenation as a bridge to recovery, is described.

\section{CASE REPORT}

A previously healthy 49-year-old Qatari woman, presented at the Communicable Disease Hospital, Doha, Qatar, with a history of cough and shortness of breath over the preceding week.

She was diagnosed with coronavirus disease 2019 (COVID-19) by real-time reverse transcription-polymerase chain reaction (RT-PCR) test, on the day of admission. Her initial chest $\mathrm{x}$-ray demonstrated bilateral infiltrates (Figure 1a) consistent with COVID-19 pneumonia. Initially, the patient had minimal respiratory symptoms. On the day of admission, after the diagnosis was made, she was prescribed $500 \mathrm{mg}$ azithromycin (Actimycin ${ }^{\mathrm{Tx}}$, Venus, India) orally once daily, for seven days, two gms ceftriaxone (Rocephin ${ }^{\mathrm{Tm}}$, Fresenius, Portugal) intravenous once daily, for three days, $400 \mathrm{mg}$ hydroxychloroquine (Paquenil ${ }^{\text {tw }}$, Sanofi, India) orally once daily for ten days, and $150 \mathrm{mg}$ oseltamivir (Tamiflue $^{\mathrm{rx}}$, Roche, Switzerland) orally twice daily, for five days. This combination of drugs corresponded with the hospital protocol. Also, low molecular weight heparin $40 \mathrm{mg}$ enoxaparin (Clexane ${ }^{\mathrm{rn}}$, Sanofi, India) subcutaneous once daily for eight days, was prescribed as a prophylaxis for deep venous thrombosis.

On Day 2, the patient became febrile, tachypneic, and had oxygen desaturation despite the escalation in non-invasive ventilation; arterial blood gas sample was consistent with Type 1 respiratory failure showing a low $\mathrm{PaO} 2 / \mathrm{FiO} 2$ ratio (Table 1 ). Repeat chest $\mathrm{X}$ rays demonstrated a worsening of the bilateral lung infiltrates (Figure 1b). A diagnosis of acute respiratory distress syndrome (ARDS) was made, and the patient was transferred to the hospital's intensive care unit for further management.

In the ICU, the patient was intubated. On Day 3, her antibiotic regime was increased to include to 4 grams piperacillin intravenous every six hours for seven days, 

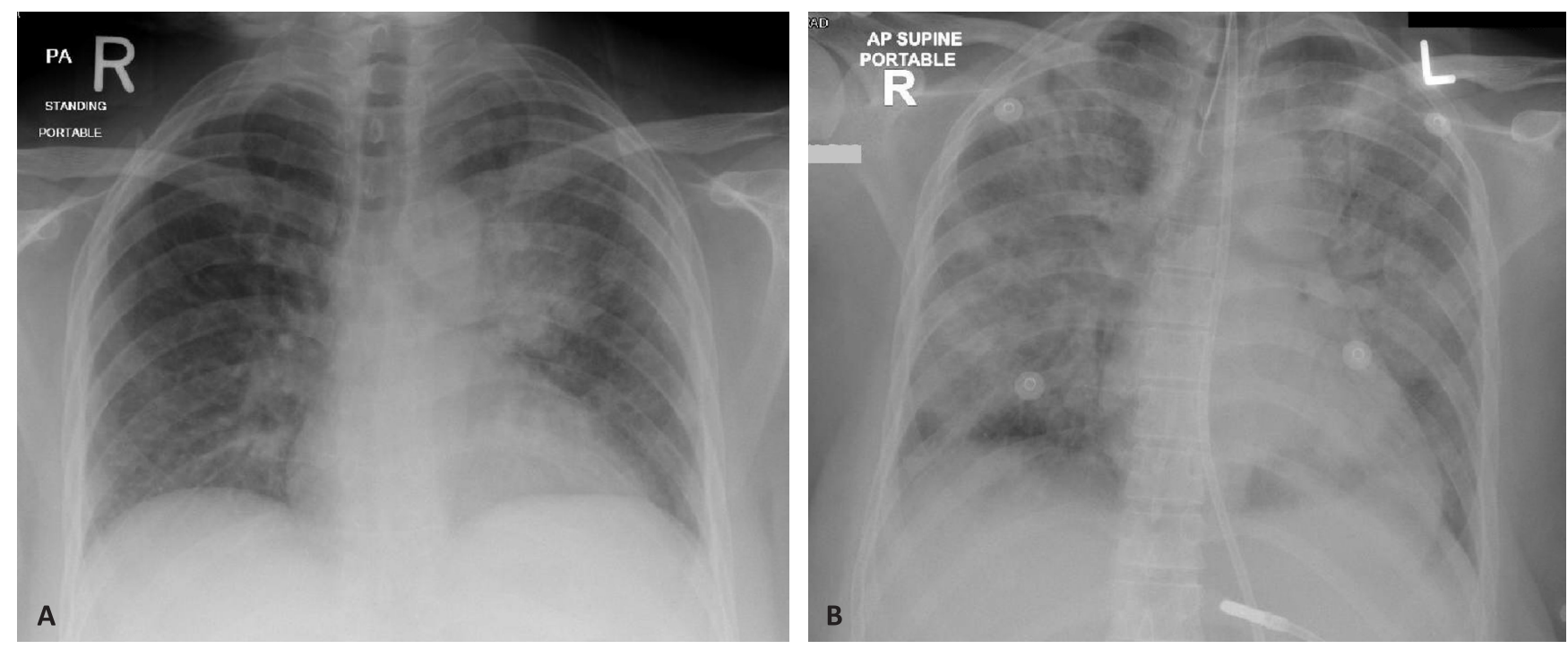

Fig. 1. A. The Initial Chest X-ray. B. The repeat Chest X-ray

in combination with $0.5 \mathrm{~g}$ tazobactam intravenous every six hours for seven days Tazocin $^{\mathrm{Tm}}$, Wyeth, Italy). Also, initially, intravenously $600 \mathrm{mg}$ teicoplanin (Targocid $^{\text {tw }}$, Sanofi, Italy) was prescribed twice daily for two days after which the dose was reduced to $400 \mathrm{mg}$ intravenously once daily for eight days to cover for hospitalacquired bacterial infections.

Given the concern that a cytokine storm was the possible the aetiology underlying the patient deteriora- tion, on Day 3, 40 mg intravenous methylprednisolone (Depo-medrol ${ }^{\mathrm{Tm}}$, Pfizer, Belgium), was administered twice daily for twelve days, together with $400 \mathrm{mg}$ intravenous tocilizumab (Actemra ${ }^{\text {rix }}$, Roche, Switzerland).

On Day 3, she also received $400 \mathrm{mg}$ lopinavir with $100 \mathrm{mg}$ ritonavir (Lopinavir / Ritonavir ${ }^{\mathrm{rx}}$, Mylan, India) orally twice daily for five days. _These were given in addition to the continuation of hydroxychloroquine, oseltamivir, and azithromycin.

\section{Table 1. Laboratory parameters of the patient throughout hospitalization}

\begin{tabular}{|c|c|c|c|c|c|c|c|c|c|c|c|}
\hline & Reference & $\begin{array}{l}\text { Ar- } \\
\text { rival }\end{array}$ & $\begin{array}{l}\text { Day } 2 \text { - } \\
\text { Intuba- } \\
\text { tion }\end{array}$ & D5 & D8 & $\begin{array}{l}\text { D9 - VA } \\
\text { ECMO }\end{array}$ & D11 & D12 & $\begin{array}{c}\text { D14 -VV } \\
\text { ECMO }\end{array}$ & $\begin{array}{c}\text { D16 - } \\
\text { ECMO } \\
\text { decan- } \\
\text { nulation }\end{array}$ & $\begin{array}{l}\text { Dis- } \\
\text { charge }\end{array}$ \\
\hline \multicolumn{12}{|l|}{ Laboratory Test } \\
\hline WBC & $4-10^{3} \mathrm{u} / \mathrm{L}$ & 7.8 & 11.2 & 14.9 & 33.3 & 32.2 & 26 & 33 & & & 10 \\
\hline $\begin{array}{l}\text { Absolute } \\
\text { Lymphocytes }\end{array}$ & $1-3^{3} u / L$ & 0.7 & 0.4 & & & 0.7 & & & & & \\
\hline CRP & $0-5 \mathrm{mg} / \mathrm{L}$ & 193 & 428 & & 23 & 20 & 11 & 8 & 5 & 4 & \\
\hline Procalcitonin & $<0.5 \mathrm{ng} / \mathrm{mL}$ & & 1.38 & 7.14 & 6.27 & 2.89 & 0.94 & & 0.25 & & \\
\hline Pro-BNP & $<125 \mathrm{pg} / \mathrm{mL}$ & & 430 & & 34.198 & & 4.403 & & & & 1.132 \\
\hline Troponin T & 3-10 ng/L & 9 & 146 & 112 & 1.076 & 1.401 & & & & & 75 \\
\hline Myoglobin & $25-58 \mathrm{ng} / \mathrm{mL}$ & & & 331 & & & & & & & \\
\hline Ferritin & $18-340$ ug/L & & 628 & 928 & 4.689 & 1,633 & 1.959 & 1.339 & 576 & 457 & 303 \\
\hline $\mathrm{LDH}$ & $135-214 \mathrm{U} / \mathrm{L}$ & & 548 & 835 & 1.239 & 980 & 948 & 1,011 & $>1000$ & 1249 & \\
\hline AST/ALT & $<33 \mathrm{U} / \mathrm{L}$ & $23 / 12$ & $35 / 15$ & $49 / 27$ & $251 / 114$ & $118 / 95$ & $82 / 97$ & $70 / 74$ & $131 / 90$ & $137 / 138$ & $23 / 32$ \\
\hline Triglycerides & $<1.7 \mathrm{mmol} / \mathrm{L}$ & & 1.4 & 2.7 & 3.1 & 1.8 & 1.1 & 1.4 & 2 & 1.8 & \\
\hline \multicolumn{12}{|l|}{ Blood Gases } \\
\hline $\mathrm{FiO}_{2}(\%)$ & & & $50 \%$ & $50 \%$ & $45 \%$ & $50 \%$ & & & $30 \%$ & $40 \%$ & \\
\hline $\mathrm{PaO}_{2}(\mathrm{mmHg})$ & & & 79 & 77 & 56 & 61 & & & 90 & 123 & \\
\hline $\mathrm{PH}$ & 7.35 to 7.45 & & 7.37 & 7.56 & 7.47 & 7.36 & & & 7.43 & 7.42 & \\
\hline Lactate & $0.5-2.2 \mathrm{mmol} / \mathrm{L}$ & & 1.4 & 2.6 & 3.4 & 5.6 & & & 1.6 & 0.8 & \\
\hline
\end{tabular}


Later, on Day 3 of hospitalization, the patient was moved to the prone position to improve her oxygenation. Initial transthoracic echocardiography, on Day 5, demonstrated preservation of left ventricular ejection fraction of $58 \%$ with no regional wall motion abnormalities. The patient was maintained on minute-ventilation with a PEEP of 10 and $\mathrm{FiO}_{2}$ of $40 \%$, to keep her blood oxygen saturation within the acceptable range.

The patient clinical condition gradually improved with decreasing ventilator support. Blood analyses showed a reduction in inflammatory markers with a drop in C-reactive protein from 428 to $23 \mathrm{mg} / \mathrm{L}$ until Day 7. On Day 7 she had a fever spike, her temperature recorded as $38.9{ }^{\circ} \mathrm{C}$, and she became hypotensive requiring initiation of vasopressors, norepinephrine (Veraline $^{\mathrm{rm}}$, Verve, India) $0.1 \mathrm{mcg} / \mathrm{kg} / \mathrm{min}$ as a continuous intravenous infusion. Peak high sensitivity Troponin $\mathrm{T}$ levels of 1,401 ng/L and Pro- BNP at 34,198 pg/mL were recorded, indicative of myocardial injury (Figure 2).

A follow-up transthoracic echocardiogram on Day 8 showed a significant reduction in the left ventricular ejection fraction to $25 \%$ with diffusely hypokinetic walls and a reduction in right ventricular function. The patient's inflammatory markers were significantly elevated (Table 1).

Given the presence of severe biventricular dysfunction on transthoracic echocardiogram, low cardiac output at $2.5 \mathrm{~L} / \mathrm{min}$ measured by pulse contour cardiac output PiCCO (Pulsion Medical Systems, Munich, Germany), elevated central venous pressure of $24 \mathrm{mmHg}$, and low central venous saturation of $50 \%$ measured from the right internal jugular central line, a diagnosis of cardiogenic shock secondary to severe myocarditis was made. Severe myocarditis was thought to be secondary to the cytokine storm syndrome. Therefore, it was decided to administer a second dose of tocilizum$\mathrm{ab}$ in addition to intravenous immunoglobulin and an additional course of pulse steroid therapy.

Organ hypoperfusion and shock physiology were refractory to the pharmacological therapy, Norepinephrine, Dobutamine, and Vasopressin, which were first prescribed on Days 7, 8 and 9 respectively. On Day 9, the patient was started on veno-arterial extracorporeal membrane oxygenation (VA-ECMO) and continuous venous-venous haemodiafiltration using CytoSorb (LINC Medical Systems Ltd., Leicester, UK.) therapy, with the aim to reduce the levels of cytokines in the blood.

On Day 10, a sub-segmental pulmonary embolism was confirmed by computed tomography, (Figure 3) and treated with a continuous intravenous infusion of heparin at 1040 unit/ per hour (16 unit $/ \mathrm{kg} / \mathrm{hr}$ ).

On Day 14, the patient's arterial ECMO access was de-cannulated, and she was converted to venous-venous ECMO to provide respiratory support only.

A follow-up transthoracic echocardiogram showed a left ventricular ejection fraction of $26 \%$ with an improvement of cardiac output. The patient's blood lactate levels normalized, and she was weaned of inotropic

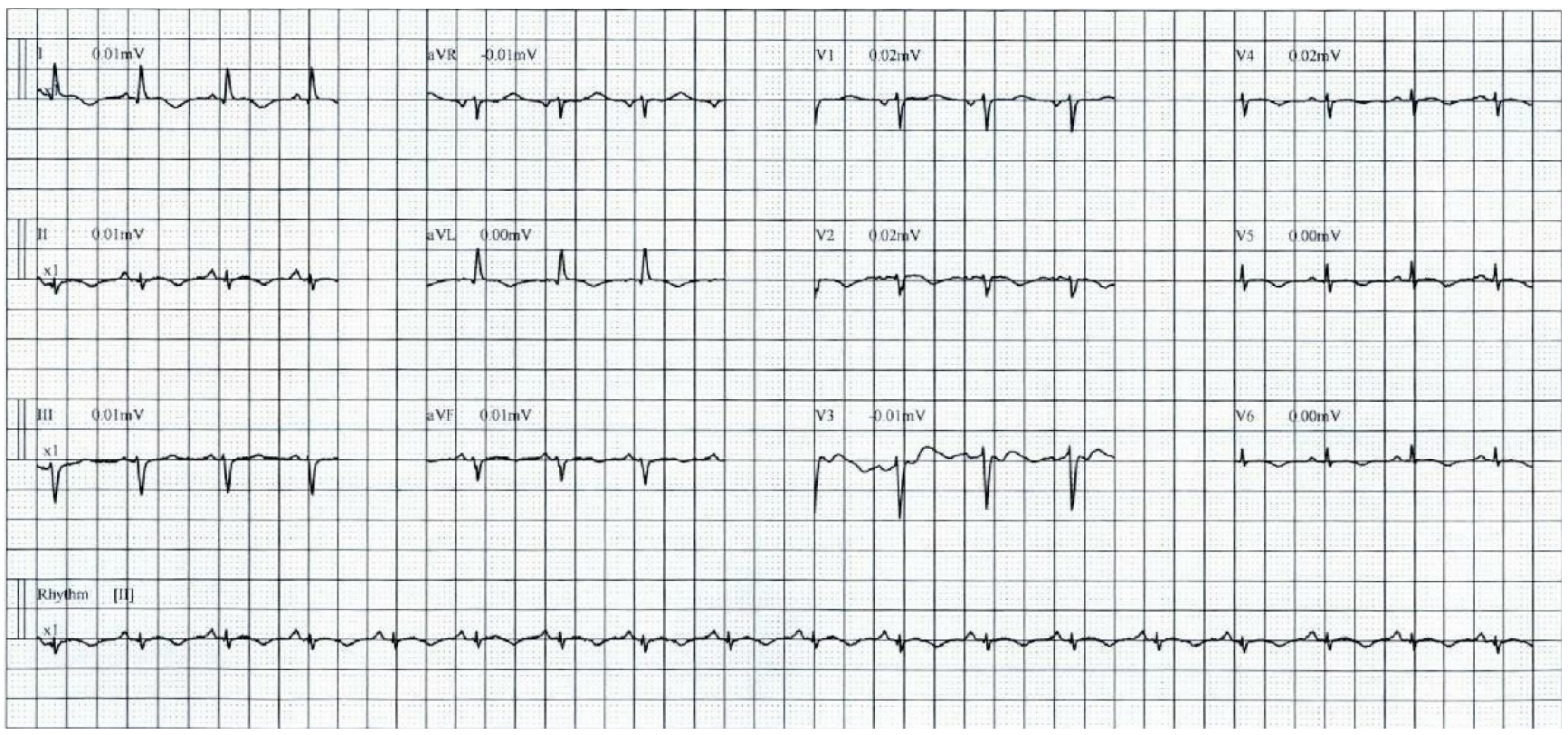

Fig. 2. ECG taken on Day 8 showed T wave inversion in the lateral leads. 


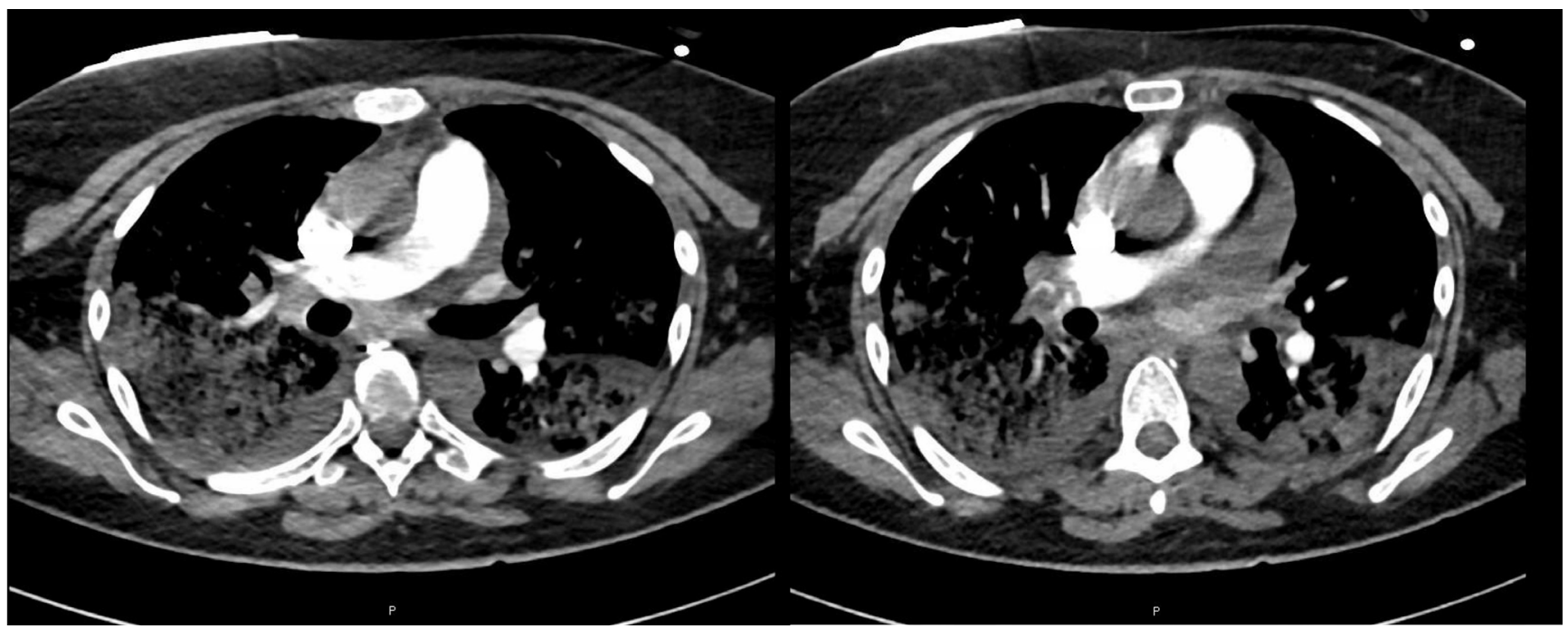

Fig. 3 CT chest revealing a pulmonary embolism

support. She was de-cannulated from VV ECMO on Day 16 and was successfully extubated on Day 23.

The patient's condition improved gradually, off oxygen, and a repeat transthoracic echocardiogram at 60 days showed improved cardiac function with a left ventricular ejection fraction of $50 \%$.

At the time of reporting this case, she was actively undergoing rehabilitation for critical illness-induced myopathy.

\section{DISCUSSION}

A case of COVID-19 infection resulting in severe ARDS followed by myocarditis with ensuing cardiogenic shock managed with VA ECMO support is reported. At the time of writing this case, a survey of the literature indicated that this is the first COVID-19 myocarditis reported from the Middle East and $12^{\text {th }}$ reported patient worldwide (Table 2). The first reported patient with COVID-19 myocarditis was from China [2]. Since then, ten more cases $[3-11,15,16]$ were reported worldwide. The age range of the patients was two to sixty-nine years. The patient's symptoms varied from being asymptomatic to presenting with typical angina symptoms and shock. The reported ECGs, at presentation, were also variable with changes consistent of T-elevation myocardial infarction. Few of the patients had undergone a coronary evaluation to exclude obstructive coronary artery disease. The diagnosis of myocarditis in most of the cases was based on clinical grounds. Cardiovascular magnetic resonance imaging (CMR) was used in some cases to confirm the diagnosis, and three patients underwent myocardial biopsies $[6,11,15,16]$. Sala et al. (2020) [6] reported a forty-three year old Italian woman who presented with a three-day history of oppressive chest pain and dyspnoea; her past medical history was unremarkable. An electrocardiogram (ECG) showed an ST-segment elevation in leads V1-2 and a VR with reciprocal ST depression. There was a reduction in myocardial function as seen on the transthoracic echocardiogram with an elevation of myocardial enzymes. Cardiovascular magnetic resonance imaging showed features consistent with Takotsubo cardiomyopathy and a myocardial biopsy demonstrated diffuse T-lymphocytic inflammatory infiltrates with interstitial oedema and limited foci of necrosis. There was an absence of the SARS-CoV-2 genome within the myocardium. Ruihai Zhou (2020) [12] argued that the patient might have had Takotsubo cardiomyopathy rather than myocarditis because of the lack of evidence of myocardial viral genome and the shared CMR features with Takotsubo cardiomyopathy.

More recently, Kesci et al. (2020) reported COVID-19 RT-PCR positivity in the cardiac tissue of a twoyear old Turkish boy with COVID-19 myocarditis; the patient also required ECMO mechanical support for management [11].

The localization of viral particles in cardiac tissue of a patient with COVID-19 cardiogenic shock was subsequently reported by Tavazzi et al. (2020), who stated that they were mainly present in cytopathic and structurally damaged interstitial cells [15].

In our case, the presence of global hypokinesia with the absence of typical echocardiographic characteristics, ECG features of Takotsubo cardiomyopathy, the sudden onset of cardiogenic shock and the significant 


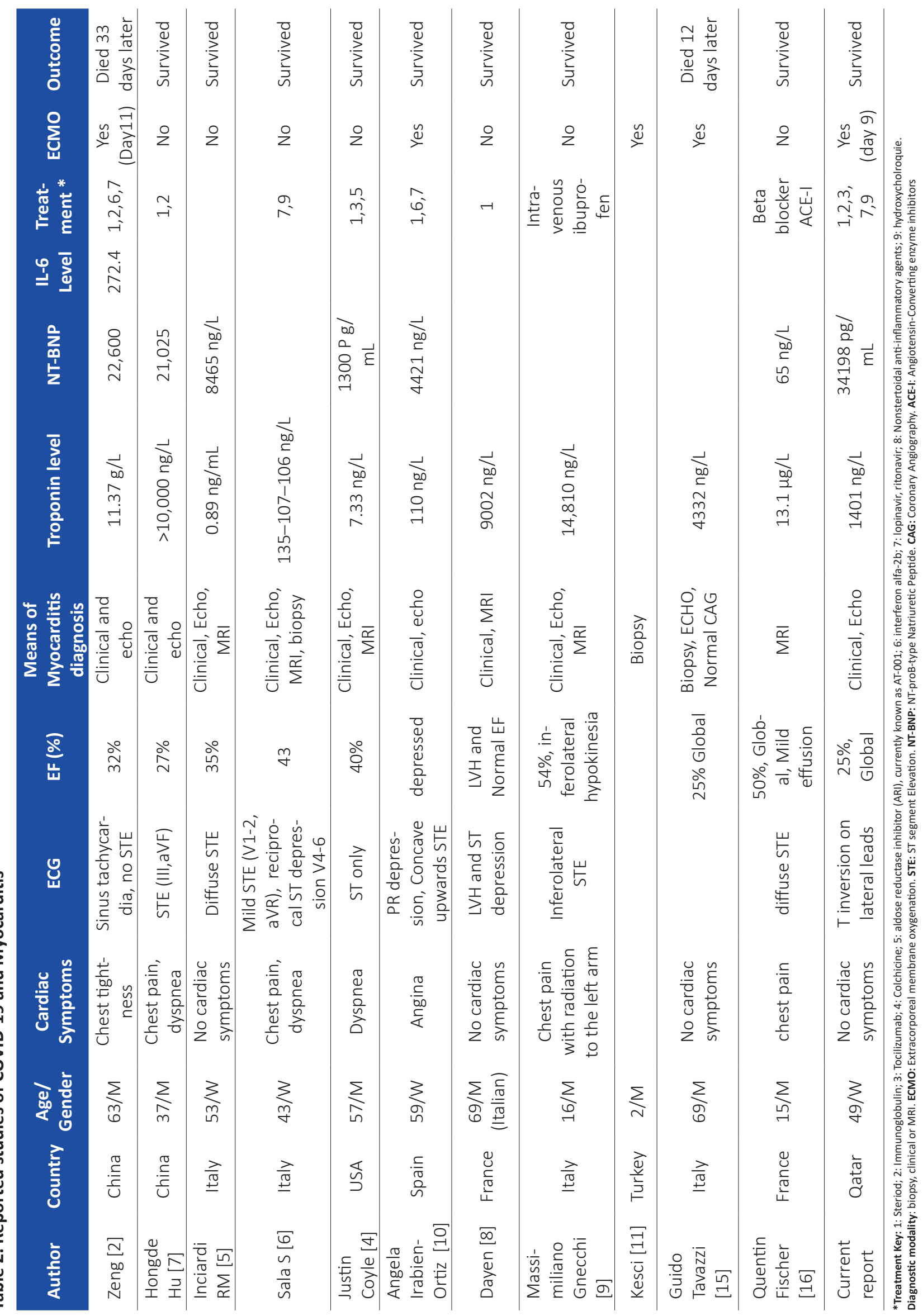


rise in cardiac markers, supported the diagnosis of acute myocarditis.

The exact mechanism of cardiac involvement in COVID-19 remains under investigation [17]. One potential mechanism is direct myocardial involvement mediated by angiotensin-converting enzyme ACE2. Other suggested mechanisms of COVID-19-related cardiac involvement include a cytokine storm, mediated by an imbalanced response among subtypes of $\mathrm{T}$ helper cells, and hypoxia-induced excessive intracellular calcium leading to cardiac myocyte apoptosis [13]. Our patient suffered from an acute pulmonary embolism, in the absence of obvious secondary cause, which we think is linked to the hypercoagulable state related to COVID-19.

At the time of writing this case, the only therapy that had shown improved survival in severe intubated COVID-19 patients was systemic steroids [14]. The role of intervention with mechanical circulatory support in COVID-19 cases is also unclear.

In the present case, the medical team deemed that the patient would not survive without mechanical circulatory support due to her continuing deteriorate, despite escalating doses of inotropes and vasopressors. Given that both lung and cardiac function were deteriorating, VA ECMO was the preferred choice of mechanical circulatory support.

The use of ECMO has been reported in four COVID-19 patients $[2,10,11,15]$, two of them died from secondary infections $[2,15]$, and the other two patients recovered $[10,11]$. Although the clinical outcome of patients with COVID-19 myocarditis is unknown, it should be noted that only two out of eleven reported patients died due to secondary infections.

\section{CONCLUSION}

Myocarditis is a rare complication of COVID-19 infection that may result in cardiogenic shock and death. In severe cases, initiation of mechanical circulatory support might be vital as a bridge to complete recovery.

\section{CONFLICT OF INTEREST}

None to declare.

\section{ETHICAL APPROVAL}

The Ethics Committee approved the study, 6/2020 (MRC-05-113). Written informed consent was ob- tained from the patient for publication of this case and any accompanying images.

\section{REFERENCES}

1. Hendren NS, Drazner MH, Bozkurt B, Cooper LT Jr. Description and Proposed Management of the Acute COVID-19 Cardiovascular Syndrome. Circulation. 2020;141(23):1903-14.

2. Zeng JH, Liu YX, Yuan J, et al. First case of COVID-19 complicated with fulminant myocarditis: a case report and insights. Infection. 2020;1-5. doi:10.1007/s15010-020-014245. [Epub ahead of print]

3. Paul JF, Charles P, Richaud C, Caussin C, Diakov C. Myocarditis revealing COVID-19 infection in a young patient. Eur Heart J Cardiovasc Imaging. 2020;21(7):776.

4. Coyle J, Igbinomwanhia E, Sanchez-Nadales A, Danciu S, Chu C, Shah N. A Recovered Case of COVID-19 Myocarditis and ARDS Treated with Corticosteroids, Tocilizumab, and Experimental AT-001. JACC Case Rep. 2020;2(9):1331-6. doi:10.1016/j. jaccas.2020.04.025. [Epub ahead of print]

5. Inciardi RM, Lupi L, Zaccone G, et al. Cardiac Involvement in a Patient With Coronavirus Disease 2019 (COVID-19). JAMA Cardiol. 2020;5(7):1-6. doi:10.1001/jamacardio.2020.1096. [Epub ahead of print]

6. Sala S, Peretto G, Gramegna M, et al. Acute myocarditis presenting as a reverse Tako-Tsubo syndrome in a patient with SARS-CoV-2 respiratory infection. Eur Heart J. 2020;41(19):1861-2.

7. Hu H, Ma F, Wei X, Fang Y. Coronavirus fulminant myocarditis saved with glucocorticoid and human immunoglobulin. Eur Heart J. 2020;ehaa190. doi:10.1093/eurheartj/ehaa190. [Epub ahead of print]

8. Doyen D, Moceri P, Ducreux D, Dellamonica J. Myocarditis in a patient with COVID-19: a cause of raised troponin and ECG changes. Lancet. 2020;395(10235):1516.

9. Gnecchi M, Moretti F, Bassi EM, et al. Myocarditis in a 16-year-old boy positive for SARS-CoV-2. Lancet. 2020;395(10242):e116.

10. Irabien-Ortiz Á, Carreras-Mora J, Sionis A, Pàmies J, Montiel J, Tauron M. Fulminant myocarditis due to COVID-19. Rev Esp Cardiol (Engl Ed). 2020;73(6):503-4.

11. Kesici S, Aykan HH, Orhan D, Bayrakci B. Fulminant COVID-19related myocarditis in an infant. Eur Heart J. 2020; ehaa515. doi:10.1093/eurheartj/ehaa515. [Epub ahead of print]

12. Zhou R. Does SARS-CoV-2 cause viral myocarditis in COVID-19 patients?. Eur Heart J. 2020;41(22):2123.

13. Clerkin KJ, Fried JA, Raikhelkar J, et al. COVID-19 and Cardiovascular Disease. Circulation. 2020;141(20):1648-55.

14. Horby P, Lim WS, et al. (RECOVERY Collaborative Group) Dexamethasone in Hospitalized Patients with Covid-19 - Preliminary Report. N Engl J Med. 2020. doi:10.1056/ NEJMoa2021436 [Epub ahead of print] 
Available online at: www.jccm.ro

15. Tavazzi G, Pellegrini C, Maurelli M, et al. Myocardial localization of coronavirus in COVID-19 cardiogenic shock. Eur J Heart Fail. 2020;22(5):911-5.

16. Fischer Q, Brillat-Savarin N, Ducrocq G, Ou P. Case report of an isolated myocarditis due to COVID-19 infection in a paediatric
The Journal of Critical Care Medicine 2021;7(2) • 129

patient. Eur Heart J Case Rep. 2020. doi: 10.1093/ehjcr/ ytaa180. [Epub ahead of print]

17. Nishant R Tiwari1, Khalid I Khatib, Subhal B Dixit, et al. Anticoagulation in COVID - 19: An Update. The Journal of Critical Care Medicine 2020;6: 217-223. 